PP97-52

WISC-MILW-96-TH-26

gr-qc/9610071

\title{
Hamiltonian thermodynamics of a Lovelock black hole
}

\author{
Jorma Louko* and Jonathan Z. Simon ${ }^{\dagger}$ \\ Department of Physics, University of Maryland, College Park, Maryland 20742-4111, USA \\ Stephen N. Winters-Hilt \\ Department of Physics, University of Wisconsin-Milwaukee, \\ P.O. Box 413, Milwaukee, Wisconsin 53201, USA
}

(October 1996)

\begin{abstract}
We consider the Hamiltonian dynamics and thermodynamics of spherically symmetric spacetimes within a one-parameter family of five-dimensional Lovelock theories. We adopt boundary conditions that make every classical solution part of a black hole exterior, with the spacelike hypersurfaces extending from the horizon bifurcation three-sphere to a timelike boundary with fixed intrinsic metric. The constraints are simplified by a Kuchař-type canonical transformation, and the theory is reduced to its true dynamical degrees of freedom. After quantization, the trace of the analytically continued Lorentzian time evolution operator is interpreted as the partition function of a thermodynamical canonical ensemble. Whenever the partition function is dominated by a Euclidean black hole solution, the entropy is given by the Lovelock analogue of the Bekenstein-Hawking entropy; in particular, in the low temperature limit the system exhibits a dominant classical solution that has no counterpart in Einstein's theory. The asymptotically flat space limit of the partition function does not exist. The results indicate qualitative robustness of the thermodynamics of five-dimensional Einstein theory upon the addition of a nontrivial
\end{abstract}

\footnotetext{
*On leave of absence from Department of Physics, University of Helsinki. Electronic address: louko@wam.umd.edu

${ }^{\dagger}$ Present address: Institute for Systems Research, University of Maryland, College Park, Maryland 20742, USA. Electronic address: jzsimon@isr.umd.edu

${ }^{\ddagger}$ Electronic address: winters@csd.uwm.edu
} 
Lovelock term.

Pacs: 04.60.Ds, 04.60.Kz, 04.70.Dy, 04.20.Fy 


\section{INTRODUCTION}

A gravitational theory whose Lagrangian density consists of multiples of lowerdimensional Euler densities has the property that the field equations are second order in the metric [1,2]. These theories, known as Lovelock theories, include Einstein's theory with a cosmological constant in all dimensions greater than two, and in five or more dimensions they provide genuine curvature squared generalizations of Einstein's theory. Among all curvature squared generalizations of Einstein's theory, Lovelock theories therefore have a special status in that they preserve the number of degrees of freedom: a generic curvature squared action yields field equations that are fourth order in the metric, containing thus more degrees of freedom than Einstein's theory. This has generated wide interest in Lovelock theories, especially in the contexts of cosmology and black hole physics [3]

The purpose of the present paper is to analyze the classical and quantum dynamics of spherically symmetric Lovelock gravity by the Hamiltonian methods recently developed by Kuchař [20]. These methods have previously been applied to spherically symmetric Einstein(-Maxwell) gravity in four dimensions [20 24], vacuum dilatonic gravity in two dimensions [23,25,26], and to related systems [27,28]; for related discussion, see Refs. [29 [35]. At the classical level, we wish to find a canonical transformation that introduces the mass parameter of the spacetime as a new canonical variable, use this transformation to simplify the constraints, and reduce the theory to its true dynamical degrees of freedom. At the quantum level, we wish to derive from the quantum theory a partition function that describes the equilibrium thermodynamics of a Lovelock black hole in the canonical ensemble.

The issues of prime interest are twofold. First, although Lovelock theories have the same set of canonical variables as Einstein's theory, the Lovelock Hamiltonian is, in general, a multivalued function of the canonical variables [7,8]. One anticipates that this multivaluedness may introduce additional features in the canonical formulation and Hamiltonian reduction, even though the Lovelock analogue of Birkhoff's theorem [0] strongly suggests that the local considerations should differ little from those in Einstein's theory. Second, certain Lovelock black holes have thermodynamical properties that differ qualitatively from those of Einstein black holes; in particular, a Lovelock black hole can be stable against Hawking evaporation in asymptotically flat space [11]. This leads one to ask whether Lovelock theories might admit quantum thermodynamical ensembles with boundary conditions that do not give rise to well-defined ensembles in Einstein's theory.

The number of possible Lovelock terms in the action increases with the dimension of the spacetime, and different choices for the coefficients yield qualitatively different theories. In this paper we shall aim for concreteness at the expense of generality. We concentrate on a specific one-parameter family of Lovelock theories exhibiting both a multivalued Hamiltonian and asymptotically flat black hole solutions that are stable against Hawking evaporation.

We take the only bulk contributions to the action to be the Einstein-Hilbert term and the four-dimensional Euler density. In $D$ spacetime dimensions, the action then reads [11]

$$
\begin{aligned}
S= & \frac{1}{2 \kappa} \int d^{D} x \sqrt{-g}\left[R+\frac{\lambda}{2}\left(R_{a b c d} R^{a b c d}-4 R_{a b} R^{a b}+R^{2}\right)\right] \\
& + \text { (boundary terms) }
\end{aligned}
$$


where $\kappa$ is the $D$-dimensional gravitational constant and $\lambda$ is the single Lovelock parameter. For $D \geq 5$, the four-dimensional Euler density contributes to the equations of motion, and we obtain a one-parameter family of generalizations of Einstein's theory. In these theories, asymptotically flat black hole solutions that are stable against Hawking evaporation occur only when $D=5$ and $\lambda>0$ [11], and we shall therefore concentrate on this case. For the interest of comparison, we shall also include the limiting case of five-dimensional Einstein theory, $D=5$ and $\lambda=0$.

We shall formulate the spherically symmetric Hamiltonian theory with thermodynamically motivated boundary conditions similar to those introduced in Ref. [21]. On a classical solution, one end of the spacelike hypersurfaces will be at the bifurcation three-sphere of a nondegenerate Killing horizon, and the other end will be on a timelike hypersurface in an exterior region of the spacetime. We shall refer to the two ends respectively as the "left" end and the "right" end, as motivated by the Penrose diagram in which our classical solutions are embedded in the right-hand-side asymptotically flat region [11]: on a solution, one can think of the left end as the inner one and the right end as the outer one. At the left end we fix the rate at which the spacelike hypersurfaces are boosted with respect to the coordinate time, and at the right end we fix the intrinsic metric on the timelike hypersurface.

For $\lambda>0$, the super-Hamiltonian $H$ turns out to be a multivalued function of the canonical variables. However, our boundary conditions are sufficient to uniquely determine $H$ near the left end of the spacelike hypersurfaces, and this solution for $H$ can then be uniquely extended to the full spacelike hypersurfaces by continuity. Our boundary conditions at the horizon thus eliminate the difficulties due to the multivaluedness of the super-Hamiltonian.

We shall find that the theory admits a natural generalization of the canonical transformation of Refs. [20,21]. The constraints become exceedingly simple, and a Hamiltonian reduction leads again to a single canonical pair of unconstrained degrees of freedom. On a classical solution, one member of the pair is the mass parameter, and its conjugate momentum is the difference of the Killing times at the left and right ends of the spacelike hypersurfaces.

After taking the curvature radius at the right end of the hypersurfaces to be timeindependent, we quantize the reduced theory by Hamiltonian methods. Following Ref. [21], we analytically continue the time evolution operator to imaginary time and take the trace, and interpret the resulting object as the partition function of a thermodynamical canonical ensemble. This ensemble describes black hole spacetimes in a spherical "box" whose size and boundary temperature are fixed.

In the special case of Einstein's theory, $\lambda=0$, we find that the thermodynamical properties of the system are highly similar to those of the corresponding system in four dimensions 21, 36, 37]. For high boundary temperatures, the partition function is dominated by a black hole that fills most of the box. For low boundary temperatures, on the other hand, there is no dominant classical solution. One can argue that the behavior of the partition function suggests a topological phase transition from a black hole to "hot flat space" [36, 37].

\footnotetext{
${ }^{1}$ We have set $c=\hbar=1$ : the gravitational constant $\kappa$ has the dimension of (length) ${ }^{D-2}$, and the Lovelock parameter $\lambda$ has the dimension of (length) ${ }^{2}$.
} 
For $\lambda>0$, the partition function displays several qualitatively different regions depending on the relative magnitudes of the box, the temperature, and $\lambda$. In the high temperature limit, with the other two parameters fixed, the partition function is again dominated by a classical black hole solution that fills most of the box. In the low temperature limit, with the other two parameters fixed, the partition function is now also dominated by a black hole solution: this black hole is small compared with the box, and it has no analogue in Einstein's theory. However, if $\lambda$ is small compared with the size of the box, the existence of the new dominating solution at low temperatures only has a minor effect on the behavior of the partition function. In this sense, we can say that the qualitative thermodynamical behavior of the pure Einstein system is stable against the addition of the Lovelock parameter.

When the size of the box is taken to infinity, the partition function does not have a well-defined limit, neither for $\lambda=0$ nor for $\lambda>0$. For $\lambda=0$ this is not surprising: just as in four dimensions, it reflects the fact that a Schwarzschild hole in asymptotically flat space is not stable against Hawking evaporation [36, 37]. For $\lambda>0$, on the other hand, the theory does admit asymptotically flat black hole solutions that are stable against Hawking evaporation [11, and one might therefore have expected the infinite box limit to exist. The reason why this is not the case becomes apparent when one tries to repeat our analysis with boundary conditions that replace the right-hand-side timelike boundary by an asymptotically flat infinity. The classical reduction and the construction of a quantum theory proceed without difficulty, but the effective Euclidean action of the system turns out to be unbounded below, and the formal integral expression for the partition function is divergent. The effective action has a local minimum, corresponding to the black hole that is stable against Hawking evaporation [38], but this is not sufficient to ensure the existence of the full canonical ensemble. Another system with locally thermodynamically stable classical solutions but no well-defined canonical ensemble is four-dimensional Einstein-Maxwell theory with fixed charge in asymptotically flat space 24].

The rest of the paper is as follows. In section [1] we introduce the notation and present the Hamiltonian formulation in the metric variables. In section III we perform a canonical transformation to Kuchař-type variables and reduce the theory to its unconstrained Hamiltonian degrees of freedom. The reduced theory is quantized and the partition function constructed in section IV, and the thermodynamics is analyzed in section $\mathrm{V}$. Section V1 presents a brief summary and discussion. Appendix A reviews briefly the classical solutions to the theory. The classical and quantum mechanical analyses under asymptotically flat boundary conditions are outlined in appendix B.

For the rest of paper, we shall use Planckian units that have been tailored for numer-

ical convenience: we set $c=\hbar=1$ and $\kappa=6 \pi^{2}$. All dimensionful constants have thus disappeared.

\section{METRIC FORMULATION}

In this section we introduce the model and present the Hamiltonian formulation in the metric variables.

We begin with the general five-dimensional spherically symmetric Arnowitt-Deser-Misner (ADM) metric, 


$$
d s^{2}=-N^{2} d t^{2}+\Lambda^{2}\left(d r+N^{r} d t\right)^{2}+R^{2} d \Omega_{3}^{2} .
$$

Here $d \Omega_{3}^{2}$ is the metric on the unit three-sphere, and $N, N^{r}, \Lambda$, and $R$ depend on the coordinates $t$ and $r$ only. The coordinate $r$ has the range $0 \leq r \leq 1$; this is convenient in view of our boundary conditions, which will make the radial proper distance on the constant $t$ hypersurfaces finite. Unless otherwise stated, we assume both the spatial metric and the spacetime metric to be nondegenerate. In particular, we take $\Lambda, R$, and $N$ to be positive.

Inserting the metric (2.1) in the Lovelock action (11.1) with $D=5$, integrating over the three-sphere, and dropping a total derivative, we recover the action

$$
S_{\Sigma}^{L}=\int d t \int_{0}^{1} d r \mathcal{L}
$$

where

$$
\begin{aligned}
\mathcal{L}= & -\frac{\left[\dot{\Lambda}-\left(N^{r} \Lambda\right)^{\prime}\right]\left(\dot{R}-N^{r} R^{\prime}\right)}{N}\left\{R^{2}+\hat{\lambda}\left[1-\left(\frac{R^{\prime}}{\Lambda}\right)^{2}+\frac{\left(\dot{R}-N^{r} R^{\prime}\right)^{2}}{3 N^{2}}\right]\right\} \\
& -\frac{\left(\dot{R}-N^{r} R^{\prime}\right)^{2}}{N}\left[\Lambda R-\hat{\lambda}\left(\frac{R^{\prime}}{\Lambda}\right)^{\prime}\right] \\
& +N \Lambda R\left[1-\left(\frac{R^{\prime}}{\Lambda}\right)^{2}\right]-N\left(\frac{R^{\prime}}{\Lambda}\right)^{\prime}\left\{R^{2}+\hat{\lambda}\left[1-\left(\frac{R^{\prime}}{\Lambda}\right)^{2}\right]\right\} .
\end{aligned}
$$

The overdot and the prime denote respectively $\partial /(\partial t)$ and $\partial /(\partial r)$. We have written $\lambda=\frac{1}{2} \hat{\lambda}$, conforming to the notation of Ref. [11]. We have verified that the Lagrangian equations of motion obtained from local variations of $S_{\Sigma}^{L}(2.2)$ are equivalent to the full spherically symmetric Lovelock equations [3 5] derived from the action (1.1). The reduction of the action by spherical symmetry is therefore consistent with the equations of motion, and we can take $S_{\Sigma}^{L}(2.2)$ as the starting point of the dynamical analysis. We shall address the boundary conditions and boundary terms within the Hamiltonian formulation below.

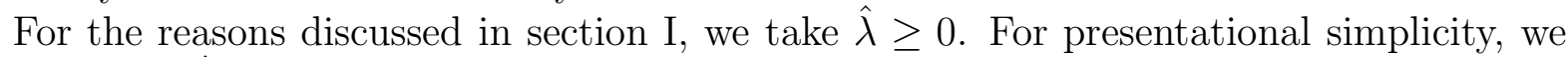
shall assume $\hat{\lambda}>0$ until explicitly stated otherwise in section $\nabla$. In the limiting case of five-dimensional Einstein gravity, $\hat{\lambda}=0$, the analysis would proceed in an entirely analogous manner, with the obvious technical simplifications.

The Hamiltonian form of the action (2.2) is

$$
S_{\Sigma}=\int d t \int_{0}^{1} d r\left(P_{\Lambda} \dot{\Lambda}+P_{R} \dot{R}-N H-N^{r} H_{r}\right),
$$

where the super-Hamiltonian constraint and the supermomentum constraint are given respectively by

$$
\begin{aligned}
H= & y\left\{P_{R}+y\left[\Lambda R-\hat{\lambda}\left(\frac{R^{\prime}}{\Lambda}\right)^{\prime}\right]\right\} \\
& -\Lambda R\left[1-\left(\frac{R^{\prime}}{\Lambda}\right)^{2}\right]+\left(\frac{R^{\prime}}{\Lambda}\right)^{\prime}\left\{R^{2}+\hat{\lambda}\left[1-\left(\frac{R^{\prime}}{\Lambda}\right)^{2}\right]\right\}, \\
H_{r}= & R^{\prime} P_{R}-\Lambda P_{\Lambda}^{\prime} .
\end{aligned}
$$


The quantity $y$ is determined in terms of the canonical variables by the cubic equation

$$
0=\frac{1}{3} \hat{\lambda} y^{3}+y\left\{R^{2}+\hat{\lambda}\left[1-\left(\frac{R^{\prime}}{\Lambda}\right)^{2}\right]\right\}+P_{\Lambda} .
$$

Note that the form of the supermomentum (2.5b) is completely determined by the fact that it must generate spatial diffeomorphisms in all the canonical variables, together with the observation that $R$ and $P_{\Lambda}$ are spatial scalars whereas $\Lambda$ and $P_{R}$ are spatial densities [20].

Depending on the values of $\Lambda, R$, and $P_{\Lambda}$, the cubic (2.6) can have up to three real solutions for $y$. The super-Hamiltonian is therefore a potentially multivalued function of the canonical variables. Such multivaluedness arises generically in Lovelock theories, owing to the presence of kinetic terms higher than quadratic in the velocities in the Lagrangian density [7,8]; in our case, the highest kinetic terms in (2.3) are quartic in the velocities. We shall address this phenomenon in more detail below. The geometrical meaning of $y$ is revealed by observing that the equation of motion obtained by varying $S_{\Sigma}$ (2.4) with respect to $P_{R}$ reads

$$
\dot{R}=N y+N^{r} R^{\prime} .
$$

On a classical solution, $y$ is therefore uniquely determined by the embedding of the spacelike hypersurface in the spacetime. Conversely, when multiple real solutions to (2.6) exist, it can be verified that they generically lead to different spacetimes.

Let us turn to the boundary conditions. From the Lovelock generalization of Birkhoff's theorem [5] it follows that the local properties of the classical solutions are completely characterized by a discrete binary parameter and a continuous, mass-like parameter. The general solution is shown in curvature coordinates in appendix $A$. We wish to concentrate on the black hole solutions, whose global structure is similar to that of Kruskal manifold [11]. We further wish to attach the left end of our spacelike hypersurfaces to the bifurcation three-sphere, and to prescribe there the rate at which the hypersurfaces are boosted with respect to our coordinate time. The right end of the hypersurfaces will then be in the righthand-side exterior region, and we wish to prescribe the metric on the timelike hypersurface that this end traces. We must now specify boundary conditions and boundary terms that achieve this.

Consider first the left end of the hypersurfaces. Following the analogous treatment in Refs. 21,23,24,26], we adopt at $r \rightarrow 0$ the falloff

$$
\begin{aligned}
\Lambda(t, r) & =\Lambda_{0}(t)+O\left(r^{2}\right), \\
R(t, r) & =R_{0}(t)+R_{2}(t) r^{2}+O\left(r^{4}\right), \\
P_{\Lambda}(t, r) & =O\left(r^{3}\right) \\
P_{R}(t, r) & =O(r) \\
N(t, r) & =N_{1}(t) r+O\left(r^{3}\right), \\
N^{r}(t, r) & =N_{1}^{r}(t) r+O\left(r^{3}\right),
\end{aligned}
$$

where $\Lambda_{0}$ and $R_{0}$ are positive, and $N_{1} \geq 0 . O\left(r^{n}\right)$ stands for a term that is bounded at $r \rightarrow 0$ by a constant times $r^{n}$, and whose derivatives fall off accordingly. As in Refs. [21,23, 24, 26], 
these conditions guarantee that the classical solutions have a bifurcate horizon, they put the left end of the spacelike hypersurfaces at the bifurcation three-sphere, and they are consistent with the constraints and preserved by the Hamiltonian evolution. They also ensure that the cubic (2.6) has a unique real solution for $y$ near $r=0$. On a classical solution, the future unit normal vector $n^{a}(t)$ to the spacelike hypersurfaces at $r=0$ then evolves according to

$$
n^{a}\left(t_{1}\right) n_{a}\left(t_{2}\right)=-\cosh \left(\int_{t_{1}}^{t_{2}} \Lambda_{0}^{-1}(t) N_{1}(t) d t\right) .
$$

Next, consider the boundary conditions in the variational principle. At $r=0$, we follow Refs. [21,23, 24, 26] and make $N_{1} / \Lambda_{0}$ a prescribed function of $t$. By (2.9), this means fixing the rate at which the constant $t$ hypersurfaces are boosted at $r=0$. At $r=1$, we make $R$

and $-g_{t t}=N^{2}-\left(\Lambda N^{r}\right)^{2}$ prescribed positive-valued functions of $t$. This means fixing the intrinsic metric on the three-surface $r=1$, and in particular fixing this metric to be timelike.

Finally, we need the boundary terms to be added to the bulk action (2.4). As in Ref. [21], it can be verified that the appropriate term at $r=0$ is

$$
\int d t R_{0}\left(\frac{1}{3} R_{0}^{2}+\hat{\lambda}\right)\left(N_{1} / \Lambda_{0}\right)
$$

and the appropriate term at $r=1$ is the integral over $t$ of

$$
\begin{aligned}
& N \Lambda^{-1} R^{2} R^{\prime}-N^{r} \Lambda P_{\Lambda}-\frac{1}{2} \dot{R}\left(R^{2}+\hat{\lambda}\right) \ln \left|\frac{N+\Lambda N^{r}}{N-\Lambda N^{r}}\right| \\
& +\hat{\lambda} N\left(\frac{R^{\prime}}{\Lambda}\right)\left[1-\frac{1}{3}\left(\frac{R^{\prime}}{\Lambda}\right)^{2}-\frac{\dot{R}\left(\dot{R}-N^{r} R^{\prime}\right)}{N^{2}}\right] \\
& -\frac{\hat{\lambda} N^{r} \Lambda}{3 N}\left[\frac{\dot{R}^{3}}{N^{2}-\left(\Lambda N^{r}\right)^{2}}+\frac{N^{r}\left(R^{\prime}\right)^{3}}{\Lambda^{2}}\right] .
\end{aligned}
$$

We have therefore arrived at a variational principle with the desired boundary conditions. The Lovelock generalization of Birkhoff's theorem guarantees that classical solutions exist, and makes possible a complete description of the solutions. One should note, however, that our Hamiltonian action does not directly reflect the split of the variables at $r=1$ into the dynamical degrees of freedom versus the boundary data. With the data on the timelike boundary, the evolution no longer forms a hyperbolic system.

\section{CANONICAL TRANSFORMATION AND HAMILTONIAN REDUCTION}

In this section we simplify the constraints by a canonical transformation and reduce the theory to unconstrained Hamiltonian variables. The treatment will closely follow Refs. [20,21,22,26].

To begin, suppose that we are given the canonical data $\left(\Lambda, R, P_{\Lambda}, P_{R}\right)$ on a spacelike hypersurface embedded in a classical solution. We wish to reconstruct from this data both the spacetime and the location of the hypersurface in the spacetime.

As we have noted above, the embedding of the hypersurface in the classical solution defines a unambiguous value of $y$ : from the equation of motion (2.7), one finds that this 
value is $y_{\text {true }}:=N^{-1}\left(\dot{R}-N^{r} R^{\prime}\right)$. To reconstruct $y_{\text {true }}$ from the canonical data, one needs to solve the cubic (2.6), which may have up to three real solutions. Near $r=0$, the falloff (2.8) guarantees that the cubic has a unique real solution, and this solution must therefore be equal to $y_{\text {true. As }} r$ increases, two spurious real solutions may appear, but it is straightforward to verify that neither of the spurious real solutions can ever be equal to $y_{\text {true }}$. Therefore, $y_{\text {true }}$ is recovered from (2.6) by choosing the unique real root near $r=0$ and following this root by continuity to all $r$. We note that, generically, neither of the spurious roots for $y$ satisfies the constraint $H=0$.

After $y=y_{\text {true }}$ has been recovered, the reconstruction proceeds in full analogy with that in Ref. [20]. The function $F$ appearing in the metric (A1) is given by

$$
F=\left(\frac{R^{\prime}}{\Lambda}\right)^{2}-y^{2}
$$

and from (A2) one finds for the mass the expression

$$
M=\frac{1}{2} R^{2}(1-F)+\frac{1}{4} \hat{\lambda}(1-F)^{2} .
$$

Finally, one finds

$$
T^{\prime}=\frac{\Lambda y}{F}
$$

which specifies the location of the hypersurface up to translations in the Killing time. This completes the reconstruction.

Next, we wish to promote the reconstruction equations into a canonical transformation, valid even when the equations of motion do not hold. Provided we stay within a sufficiently narrow neighborhood of the classical solutions, $y$ is again uniquely recovered as a function of the canonical data by taking the unique real root of (2.6) near $r=0$ and continuously following this root as $r$ increases. Computing the Poisson bracket between $M$ and $T^{\prime}$ suggests that $-T^{\prime}$ could serve as the momentum conjugate to $M$; if this holds, the new momentum conjugate to $\mathrm{R}:=R$ is fixed by the fact that the supermomentum constraint generates spatial diffeomorphisms in all the variables and must thus read $P_{M} M^{\prime}+P_{\mathrm{R}} \mathrm{R}^{\prime}$. These considerations suggest the transformation

$$
\begin{aligned}
M & :=\frac{1}{2} R^{2}(1-F)+\frac{1}{4} \hat{\lambda}(1-F)^{2}, \\
P_{M} & :=-\frac{\Lambda y}{F} \\
\mathrm{R} & :=R \\
P_{\mathrm{R}} & :=F^{-1}\left(\Lambda^{-2} R^{\prime} H_{r}-y H\right),
\end{aligned}
$$

with $F$ given by (3.1). We now need to examine whether this transformation is indeed canonical.

To proceed, we arrange the difference of the integrands in the Liouville forms as 


$$
\begin{aligned}
P_{\Lambda} \delta \Lambda+ & P_{R} \delta R-P_{M} \delta M-P_{\mathrm{R}} \delta \mathrm{R} \\
= & \delta\left[\Lambda P_{\Lambda}-\hat{\lambda} y \Lambda^{-1}\left(R^{\prime}\right)^{2}+\frac{1}{2} R^{\prime}\left(R^{2}+\hat{\lambda}\right) \ln \left|\frac{R^{\prime}+y \Lambda}{R^{\prime}-y \Lambda}\right|\right] \\
& +\left\{\left[\hat{\lambda} y \Lambda^{-1} R^{\prime}-\frac{1}{2}\left(R^{2}+\hat{\lambda}\right) \ln \left|\frac{R^{\prime}+y \Lambda}{R^{\prime}-y \Lambda}\right|\right] \delta R\right\}^{\prime} .
\end{aligned}
$$

Both terms on the right-hand side of (3.5) are well defined. Upon integration from $r=0$ to $r=1$, the second term only produces contributions from the two ends. The contribution from $r=0$ vanishes because of the falloff (2.8). The contribution from $r=1$ vanishes if $\delta R$ vanishes there. As $\delta$ should in the context of the Liouville form be understood as a time derivative, this happens when the boundary conditions fix $R$ to be independent of $t$ at $r=1$. If this is the case, we see that the difference of the Liouville forms is an exact form,

$$
\begin{aligned}
& \int_{0}^{1} d r\left(P_{\Lambda} \delta \Lambda+P_{R} \delta R\right)-\int_{0}^{1} d r\left(P_{M} \delta M+P_{\mathrm{R}} \delta \mathrm{R}\right) \\
& =\delta\left\{\int_{0}^{1} d r\left[\Lambda P_{\Lambda}-\hat{\lambda} y \Lambda^{-1}\left(R^{\prime}\right)^{2}+\frac{1}{2} R^{\prime}\left(R^{2}+\hat{\lambda}\right) \ln \left|\frac{R^{\prime}+y \Lambda}{R^{\prime}-y \Lambda}\right|\right]\right\},
\end{aligned}
$$

and the transformation is canonical.

If, on the other hand, the boundary conditions fix $R$ to be explicitly $t$-dependent at $r=1$, one cannot similarly argue that $\delta R$ would vanish at $r=1$. A As mentioned at the end of section $\mathbb{I}$, the canonical variables at $r=1$ do not cleanly split into "independent" degrees of freedom versus boundary data, and it us unclear to us what the proper attitude here should be. We shall, nevertheless, proceed to regard the transformation as canonical even when $R$ is explicitly $t$-dependent at $r=1$ : as in Refs. [21,23,26], it will be seen that no apparent inconsistency will result. From the viewpoint of thermodynamics, the case of principal interest will in any case be the one where $R$ is independent of $t$ at $r=1$.

By construction, our transformation is well defined in a sufficiently narrow neighborhood of the classical solutions. It also has a unique inverse. Equations (3.4a) and (3.4c), together with the falloff implied by (2.8), determine $F$ uniquely in terms of $M$ and R. Equations (3.1) and (3.4b), together with the fact that $\Lambda$ is by assumption positive, then determine $\Lambda$ and $y . P_{\Lambda}$ is obtained from (2.6), and $P_{R}$ finally from (3.4d).

To obtain the action in the new variables, we note that the constraint terms can be written as

$$
N H+N^{r} H_{r}=N^{M} M^{\prime}+N^{\mathrm{R}} P_{\mathrm{R}},
$$

where

$$
\begin{aligned}
N^{M} & =-N F^{-1} \Lambda^{-1} R^{\prime}-N^{r} F^{-1} \Lambda y, \\
N^{\mathrm{R}} & =N y+N^{r} R^{\prime} .
\end{aligned}
$$

\footnotetext{
${ }^{2}$ This appears to have gone unmentioned in Refs. [21,23,26].
} 
This suggests that one could take $N^{M}$ and $N^{\mathrm{R}}$ as the new independent Lagrange multipliers in the action. Examining the falloff at $r=0$ reveals, however, that fixing $N^{M}$ at $r=0$ to a value that is independent of the canonical variables is not equivalent to fixing $N_{1} \Lambda_{0}^{-1}$ to a value that is independent of the canonical variables. This difficulty can be remedied by redefining the Lagrange multipliers near $r=0$ as in Ref. [24], and the appropriate boundary terms at $r=0$ and $r=1$ can then be constructed as in Refs. [21,24,26]. After these steps, the constraints can be eliminated by a Hamiltonian reduction as in Refs. [20,21,26], and one recovers a reduced theory in a true Hamiltonian form. The steps follow the cited references so closely that we shall here omit the detail and proceed directly to the reduced action.

The reduced action reads

$$
S_{\text {red }}=\int d t(\mathbf{p} \dot{\mathbf{m}}-\mathbf{h}) .
$$

The coordinate $\mathbf{m}$ arises from the unreduced theory as the $r$-independent value that $M$ takes when the constraint $M^{\prime}=0$ holds. The momentum $\mathbf{p}$ is related to the unreduced variables by

$$
\mathbf{p}:=\int_{0}^{1} d r P_{M} .
$$

The Hamiltonian $\mathbf{h}$ is given by

$$
\begin{aligned}
\mathbf{h}= & -\mathrm{N}_{0} R_{h}\left(\frac{1}{3} R_{h}^{2}+\hat{\lambda}\right) \\
& -\left(B^{2}+\hat{\lambda}\right)\left[\sqrt{Q^{2} \mathrm{~F}+\dot{B}^{2}}+\frac{1}{2} \dot{B} \ln \left(\frac{\sqrt{Q^{2} \mathrm{~F}+\dot{B}^{2}}-\dot{B}}{\sqrt{Q^{2} \mathrm{~F}+\dot{B}^{2}}+\dot{B}}\right)\right] \\
& +\frac{1}{3} \hat{\lambda} Q^{-2}\left(Q^{2} \mathrm{~F}+\dot{B}^{2}\right)^{3 / 2},
\end{aligned}
$$

where

$$
\begin{gathered}
\mathrm{N}_{0}:=N_{1} / \Lambda_{0}, \\
R_{h}:=\sqrt{2 \mathbf{m}-\frac{1}{2} \hat{\lambda}}, \\
\mathrm{F}:=1+\frac{B^{2}}{\hat{\lambda}}\left(1-\sqrt{1+\frac{4 \mathbf{m} \hat{\lambda}}{B^{4}}}\right),
\end{gathered}
$$

and $B$ and $Q^{2}$ are respectively the values of $\mathrm{R}$ and $-g_{t t}$ at $r=1 . \quad B, Q^{2}$, and $\mathrm{N}_{0}$ are considered prescribed functions of $t$, satisfying $B>0, Q^{2}>0$, and $\mathrm{N}_{0} \geq 0$. The range of $\mathbf{m}$ is $\frac{1}{4} \hat{\lambda}<\mathbf{m}<\frac{1}{2} B^{2}+\frac{1}{4} \hat{\lambda}$, corresponding to $0<R_{h}<B$, and the range of $\mathbf{p}$ is the full real axis. $R_{h}$ is equal to $R_{0}$, and on a classical solution it equals the horizon radius.

The equation of motion for $\mathbf{m}$ implies that $\mathbf{m}$ is independent of $t$ : the value of $\mathbf{m}$ is simply the mass parameter of the classical solution. The equation of motion for $\mathbf{p}$ reflects the fact that, by (3.3), (3.4b), and (3.10), $\mathbf{p}$ is equal to the difference in the Killing times at the two ends of the spacelike hypersurface. 


\section{QUANTIZATION AND THE PARTITION FUNCTION}

In this section we quantize the reduced Hamiltonian theory and obtain a partition function as the trace of the analytically continued time evolution operator.

From now on, we take the boundary radius independent of time, $\dot{B}=0$. We also subtract from the Hamiltonian (3.11) the value that the terms arising from $r=1$ would take on flat spacetime. This subtraction does not affect the equations of motion, but it does renormalize the value of the action: it is analogous to subtracting the $K_{0}$ term in Einstein's theory [39,40]. Writing $Q:=\sqrt{Q^{2}}>0$, the new Hamiltonian is given by

$$
\mathbf{h}=Q(1-\sqrt{\mathrm{F}})\left[B^{2}+\frac{1}{3} \hat{\lambda}(1-\sqrt{\mathrm{F}})(2+\sqrt{\mathrm{F}})\right]-\mathrm{N}_{0} R_{h}\left(\frac{1}{3} R_{h}^{2}+\hat{\lambda}\right) .
$$

The first of the two terms in (4.1) is the Lovelock analogue of the quasilocal energy of Brown and York [41] 43]. The second term arises from the bifurcation three-sphere, and it will give rise to the black hole entropy.

Quantization proceeds exactly as in Refs. 21, 24, 26. We take the wave functions $\psi$ to be functions of the configuration variable $\mathbf{m}$, with $\frac{1}{4} \hat{\lambda}<\mathbf{m}<\frac{1}{2} B^{2}+\frac{1}{4} \hat{\lambda}$, and we introduce an inner product with some smooth and slowly varying weight factor. The Hamiltonian operator is taken to act by multiplication by the function $\mathbf{h}(4.1), \psi(\mathbf{m}) \mapsto \mathbf{h}(\mathbf{m}) \psi(\mathbf{m})$, and the unitary time evolution operator is easily found. We then analytically continue the arguments of the time evolution operator to imaginary values: we set $\int Q d t=-i \beta$, interpreting $\beta>0$ as the inverse temperature at the boundary, and $\int \mathrm{N}_{0} d t=-2 \pi i$, motivated by the regularity of the classical Euclidean solutions. The trace of the analytically continued time evolution operator is divergent, but we can argue as in Refs. [21,24, 26] that an acceptable renormalization is achieved by introducing a suitable regularization, dividing by the trace of the regularized identity operator, and finally eliminating the regulator. In this fashion, we obtain for the renormalized trace the manifestly well-defined expression

$$
Z(\beta ; B ; \hat{\lambda})=\left(\int_{0}^{1} \tilde{\mu} d x\right)^{-1}\left[\int_{0}^{1} \tilde{\mu} d x \exp \left(-I_{*}\right)\right],
$$

where the effective action $I_{*}$ is given byf

$$
I_{*}=\beta B^{2}(1-\sqrt{\mathrm{F}})\left[1+\frac{\hat{\lambda}}{3 B^{2}}(1-\sqrt{\mathrm{F}})(2+\sqrt{\mathrm{F}})\right]-2 \pi B^{3} x\left(\frac{1}{3} x^{2}+\frac{\hat{\lambda}}{B^{2}}\right)
$$

with

$$
\mathrm{F}=1+\frac{B^{2}}{\hat{\lambda}}\left(1-\sqrt{1+\frac{2 x^{2} \hat{\lambda}}{B^{2}}+\frac{\hat{\lambda}^{2}}{B^{4}}}\right) .
$$

We have introduced the integration variable $x=R_{h} / B$, and the smooth and slowly varying positive function $\tilde{\mu}(x)$ arose from the choice of the inner product.

\footnotetext{
${ }^{3}$ This effective action has been obtained previously [44 by the Euclidean Hamiltonian reduction method of Ref. 37.
} 
We now interpret the object $Z(\beta ; B ; \hat{\lambda})(4.2)$ as the partition function of a thermodynamical canonical ensemble describing black holes in a spherical box with curvature radius $B$ and and inverse boundary temperature $\beta$. The thermodynamical properties of this ensemble will be analyzed in the next section.

\section{THERMODYNAMICS IN THE CANONICAL ENSEMBLE}

As noted above, the partition function $Z(\beta ; B ; \hat{\lambda})(4.2)$ is manifestly well defined. Further, the form of the integral in (4.2) guarantees that the (constant volume) heat capacity, $C=\beta^{2}\left(\partial^{2}(\ln Z) / \partial \beta^{2}\right)$, is always positive (see, for example, section IV of Ref. [26]), and that the ensemble has a well-defined density of states 36 38,45. These properties support the interpretation of the partition function in terms of a genuine thermodynamical equilibrium ensemble, in spite of the fact that we arrived at the partition function via an analytic continuation and not via direct statistical mechanics arguments.

To proceed, we shall estimate the integral in (4.2) by the saddle point approximation. We shall throughout assume $\tilde{\mu}(x)$ to be so slowly varying that its precise form will not affect the saddle point analysis. We shall also assume that the action is sufficiently rapidly varying to make the saddle point approximation is justified, without attempting to explicitly state the necessary conditions; typically, it will be throughout assumed that the system is "macroscopic," $B \gg 1$.

The critical points of $I_{*}$ are at the roots of the equation

$$
\frac{\beta x}{2 \pi B}=\left(x^{2}+\frac{\hat{\lambda}}{B^{2}}\right) \sqrt{\mathrm{F}} .
$$

The critical points give precisely the Lorentzian black hole solutions whose Hawking temperature at the boundary, calculated in the usual way from the surface gravity [1] and the blueshift factor, is equal to $\beta$. The mass of the hole is $\mathbf{m}=\frac{1}{2} B^{2} x^{2}+\frac{1}{4} \hat{\lambda}$, and the value of $I_{*}$ at a critical point equals the Euclidean action of the corresponding Euclideanized black hole solution. Whenever the partition function is dominated by a critical point, we recover for the thermal energy expectation value and the entropy the results

$$
\begin{gathered}
\langle E\rangle=-\frac{\partial(\ln Z)}{\partial \beta} \approx B^{2}(1-\sqrt{\mathrm{F}})\left[1+\frac{\hat{\lambda}}{3 B^{2}}(1-\sqrt{\mathrm{F}})(2+\sqrt{\mathrm{F}})\right], \\
S=\left(1-\beta \frac{\partial}{\partial \beta}\right)(\ln Z) \approx 2 \pi B^{3} x\left(\frac{1}{3} x^{2}+\frac{\hat{\lambda}}{B^{2}}\right)=2 \pi R_{h}\left(\frac{1}{3} R_{h}^{2}+\hat{\lambda}\right),
\end{gathered}
$$

where $x$ and $\mathrm{F}$ are evaluated at the critical point. The expression (5.2b) for the entropy agrees with the result first obtained by Euclidean methods [11].

We can now extract physical information by analyzing the critical point structure of $I_{*}$ in various limits of interest in the three parameters $\hat{\lambda}, B$, and $\beta$.

As a preliminary, consider the case $\hat{\lambda}=0$, in which our Lovelock theory reduces to Einstein's theory. Although we have for presentational simplicity assumed $\hat{\lambda}>0$, it is easy to see that the partition function for Einstein's theory is correctly recovered by taking the 
limit $\hat{\lambda} \rightarrow 0$ in equations (4.2)-(4.4). In particular, (4.4) reduces to $\mathrm{F}=1-x^{2}$, and the critical point equation (5.1) reduces to

$$
\frac{\beta}{2 \pi B}=x \sqrt{1-x^{2}}
$$

The condition for critical points to exist is $\beta \leq \pi B$, and the critical points are then at $x=x_{ \pm}:=2^{-1 / 2}\left(1 \pm \sqrt{1-\pi^{-2} B^{-2} \beta^{2}}\right)^{1 / 2}$. When the critical points are distinct, $x_{+}$is a local minimum and $x_{-}$a local maximum. When $\beta^{2}<\frac{3}{4} \pi^{2} B^{2}$, the partition function gets its dominant contribution from the global minimum at $x=x_{+}$. When $\beta^{2}>\frac{3}{4} \pi^{2} B^{2}$, on the other hand, the partition function gets its dominant contribution from the vicinity of the global minimum at $x=0$. The limiting case $\beta^{2}=\frac{3}{4} \pi^{2} B^{2}$ represents a phase transition where the dominant contribution shifts from $x=x_{+}$to $x=0$ as $\beta$ increases. When the saddle point dominates, the thermal energy and entropy (5.2) take the form

$$
\begin{aligned}
\langle E\rangle & \approx B^{2}\left(1-\sqrt{1-x_{+}^{2}}\right) \\
S & \approx \frac{2}{3} \pi B^{3} x_{+}^{3}=\frac{2}{3} \pi R_{h}^{3}
\end{aligned}
$$

and the relation between the thermal energy and the mass can be written as

$$
\mathbf{m} \approx\langle E\rangle-\frac{\langle E\rangle^{2}}{2 B^{2}} .
$$

Equation (5.5) displays explicitly how the mass gets a contribution both from the thermal energy and from the gravitational binding energy associated with the thermal energy. Expectedly, the situation is closely similar to that in four-dimensional Einstein theory [36, 37,,45].

We now turn to the case $\hat{\lambda}>0$, in which $I_{*}$ always has at least one critical point.

Consider first the limit of small $\hat{\lambda}$ with fixed $B$ and $\beta$. The situation differs from that in the case $\hat{\lambda}=0$ only in that there is now one new critical point, a local minimum, at $x=2 \pi \hat{\lambda} B^{-1} \beta^{-1}+O\left(\hat{\lambda}^{2}\right)$. At the new critical point, $I_{*}=\frac{1}{4} \hat{\lambda} \beta+O\left(\hat{\lambda}^{2}\right)$. Therefore, as $\hat{\lambda} \rightarrow 0$, the partition function smoothly approaches that of Einstein's theory. In particular, when $\beta^{2}<\frac{3}{4} \pi^{2} B^{2}$, it would be straightforward to compute the first order correction in $\hat{\lambda}$ to the thermal energy and the entropy (5.4), assuming that the corrections to the saddle point approximation are small.

Consider next the small $\beta$ limit with fixed $B$ and $\hat{\lambda}$. There is only one critical point, at $x=1-\frac{1}{8} \pi^{-2}\left(B^{2}+\hat{\lambda}\right)^{-1} \beta^{2}+O\left(\beta^{4}\right)$, and this critical point is the global minimum of $I_{*}$. One can think of this critical point as the counterpart of the larger of the two critical points of the case $\hat{\lambda}=0$ : the black hole fills almost all of the box. The disappearance of the smaller critical point of the case $\hat{\lambda}=0$ is related to the fact that, for fixed $\hat{\lambda}$, the Hawking temperature of the Lovelock hole in asymptotically flat space is bounded below by $\frac{1}{4} \pi^{-1} \hat{\lambda}^{-1 / 2}$ [11]. If the saddle point approximation to the partition function remains good, the thermal energy and the entropy are given by

$$
\begin{aligned}
\langle E\rangle & \approx B^{2}+\frac{2}{3} \hat{\lambda}-\frac{1}{2} \pi^{-1} B \beta+O\left(\beta^{2}\right), \\
S & \approx 2 \pi B^{3}\left(\frac{1}{3}+\frac{\hat{\lambda}}{B^{2}}-\frac{\beta^{2}}{8 \pi^{2} B^{2}}\right)+O\left(\beta^{4}\right) .
\end{aligned}
$$


Consider next the large $\beta$ limit with fixed $B$ and $\hat{\lambda}$. There is again only one critical point, at $x=2 \pi \hat{\lambda} B^{-1} \beta^{-1} \mathrm{~F}_{0}^{1 / 2}+O\left(\beta^{-3}\right)$, where $\mathrm{F}_{0}:=1+B^{2} \hat{\lambda}^{-1}\left(1-\sqrt{1+\hat{\lambda}^{2} B^{-4}}\right)$. This critical point is the global minimum, and it has no counterpart in Einstein's theory: it corresponds to a small, "purely Lovelock," black hole. If the saddle point approximation to the partition function remains good, the thermal energy and the entropy are easily read off from (5.2) as

$$
\begin{aligned}
\langle E\rangle & \approx B^{2}\left(1-\mathrm{F}_{0}^{1 / 2}\right)\left[1+\frac{\hat{\lambda}}{3 B^{2}}\left(1-\mathrm{F}_{0}^{1 / 2}\right)\left(2+\mathrm{F}_{0}^{1 / 2}\right)\right]+O\left(\beta^{-2}\right), \\
S & \approx \frac{4 \pi^{2} \hat{\lambda}^{2} \mathrm{~F}_{0}^{1 / 2}}{\beta}+O\left(\beta^{-3}\right) .
\end{aligned}
$$

Consider then the large $\hat{\lambda}$ limit with fixed $B$ and $\beta$. There is again only one critical point, at $x=1-\frac{1}{8} \pi^{-2} \beta^{2} \hat{\lambda}^{-1}+O\left(\hat{\lambda}^{-2}\right)$, and this critical point is the global minimum. The hole is again "purely Lovelock," but it now fills almost all of the box.

Finally, consider the large $B$ limit with fixed $\hat{\lambda}$ and $\beta$. One critical point is at $x=$ $1-\frac{1}{8} \pi^{-2} B^{-2} \beta^{2}+O\left(B^{-4}\right)$. This critical point is the global minimum, and it can be regarded as the counterpart of the larger of the two critical points of the case $\hat{\lambda}=0$. If $\beta>4 \pi \hat{\lambda}^{1 / 2}$, there are in addition two other critical points, at $x=\frac{1}{4} \pi^{-1} B^{-1} \beta\left(1 \pm \sqrt{1-16 \pi^{2} \hat{\lambda} \beta^{-2}}\right)+O\left(B^{-3}\right)$. The fact that the two small critical points exist only for $\beta>4 \pi \hat{\lambda}^{1 / 2}$ is related to the abovementioned phenomenon that the Hawking temperature of our Lovelock hole in asymptotically flat space is bounded below by $\frac{1}{4} \pi^{-1} \hat{\lambda}^{-1 / 2}$ [1]. If the saddle point approximation is good, the thermal energy and the entropy are obtained by replacing both $O$-terms in (5.6) by $O\left(B^{-1}\right)$.

We therefore see that for $\hat{\lambda}>0$, the partition function is always dominated by a black hole solution in the limits that we have considered. In the high temperature limit and in the large box limit, the situation is very similar to that for $\hat{\lambda}=0$, in that the dominating black hole solution fills most of the box. In the low temperature limit, on the other hand, the Lovelock theory does exhibit a dominating black hole solution where none existed in the case $\hat{\lambda}=0$. For a macroscopic box and $\hat{\lambda} \ll B^{2}$, however, the presence of the new dominating solution does not appear to make the thermodynamical behavior qualitatively different from that in the case $\hat{\lambda}=0$. One can read these results as evidence for stability of the qualitative thermodynamical behavior of Einstein's theory upon the addition of the Lovelock parameter.

For $\hat{\lambda}>0$, the critical point structure of $I_{*}$ is entirely determined by two parameters, which can be conveniently taken to be $\hat{\lambda} B^{-2}$ and $\beta B^{-1}$. Numerical experimentation suggests that there are never more than three critical points. When $\hat{\lambda} B^{-2}$ is fixed and sufficiently large, there is only one critical point for any $\beta B^{-1}$ : this critical point is the global minimum, and it migrates smoothly from the large Lovelock hole to the small Lovelock hole as $\beta B^{-1}$ increases. When $\hat{\lambda} B^{-2}$ is fixed and sufficiently small, on the other hand, the transition between the unique minima for small and large $\beta B^{-1}$ takes place via a phase in which $I_{*}$ has three critical points, a maximum surrounded by two minima: as $\beta B^{-1}$ tends to zero (infinity), only the larger (smaller) of the two minima prevails. When three critical points 
exist, one can find regions of the parameter space where the global minimum is at either of the two local minima. We have, however, not attempted to corroborate these numerical experiments analytically.

It should be emphasized that the partition function has no well-defined limit as $B \rightarrow \infty$ with fixed $\beta$ and $\hat{\lambda}$, neither for $\hat{\lambda}=0$ nor for $\hat{\lambda}>0$. As with Einstein's theory in four dimensions [36,37, this reflects the fact that the thermodynamical canonical ensemble is not well defined in asymptotically flat space. We shall give a more detailed comparison of the boxed Lovelock theory to Lovelock theory in asymptotically flat space in appendix $\mathbb{B}$.

\section{SUMMARY AND DISCUSSION}

In this paper we have investigated the Hamiltonian dynamics and thermodynamics of five-dimensional spherically symmetric Lovelock theories in which the only contributions to the Lagrangian density are the Einstein-Hilbert term and the four-dimensional Euler density. We adopted boundary conditions that enforce every classical solution to be part of the exterior region of a black hole, with the spacelike hypersurfaces extending from the horizon bifurcation three-sphere to a timelike boundary with fixed intrinsic metric. We simplified the constraints by a canonical transformation that generalizes the one introduced by Kuchař in four-dimensional spherically symmetric Einstein theory, and we reduced the theory classically to its true dynamical degrees of freedom.

After Hamiltonian quantization, we interpreted the trace of the analytically continued time evolution operator as the partition function of a thermodynamical canonical ensemble, describing black holes in a spherical box whose size and boundary temperature are fixed. In the special case where the Lovelock parameter $\lambda$ vanishes and the theory reduces to Einstein's theory, we found that the thermodynamics is highly similar to that of the corresponding system in four-dimensional Einstein theory: in particular, for high boundary temperatures the partition function is dominated by a classical black hole solution that fills most of the box. When $\lambda>0$, the situation was more versatile. In the high temperature limit, with $\lambda$ and the box size fixed, the partition function is again dominated by a black hole that fills most of the box. In the low temperature limit, on the other hand, the partition function is now also dominated by a black hole solution; this black hole is small, and it has has no analogue in Einstein theory. Nevertheless, if $\lambda$ is small compared with the size of the box, the new dominating solution has little qualitative effect on the thermodynamical properties. In this sense, the qualitative thermodynamical behavior of the Einstein system is stable upon the addition of the Lovelock parameter.

When the box size is taken to infinity, we found that the partition function has no welldefined limit, neither for $\lambda=0$ nor for $\lambda>0$. While this is not surprising for Einstein's theory, in view of the similar phenomenon in four dimensions [36, 37], one might have hoped the theory with $\lambda>0$ to fare better on the grounds that this theory admits asymptotically flat black hole solutions that are stable against Hawking evaporation [11]. However, even though a classical solution that dominates a well-defined partition function must be stable against Hawking evaporation [38], our Lovelock theory in asymptotically flat space provides an example where the mere existence of such a locally stable classical solution does not imply the existence of well-defined canonical ensemble. Another such example occurs in 
four-dimensional Einstein-Maxwell theory in asymptotically flat space [24].

In the classical theory with $\lambda>0$, we saw that the super-Hamiltonian emerges as a multivalued function of the canonical variables, as is generically the case in Lovelock theories [7]:8]. Nevertheless, our thermodynamically motivated boundary conditions were sufficient to uniquely specify the super-Hamiltonian near the horizon, and the uniqueness could be extended to the full spacelike hypersurfaces by continuity. Another boundary condition that would uniquely specify the super-Hamiltonian in this fashion is the asymptotically flat falloff (B1) discussed in appendix B. However, one expects there to exist boundary conditions of interest for which such uniqueness does not occur, and in such cases one would need to seek other criteria for specifying the super-Hamiltonian. If one regards the Lovelock theory as a perturbation to Einstein's theory, or as a toy model for semiclassical gravity with backreaction, one possible criterion of this kind is perturbative expandability of the solutions in $\lambda$ [46].

In conclusion, our results provide evidence for robustness of the classical Hamiltonian structure and the qualitative thermodynamical structure of spherically symmetric Einstein gravity in five dimensions upon the addition of the four-dimensional Euler density in the action. To put this conclusion in proper perspective, one should remember that both our particular Lovelock theory and our boundary conditions were hand-picked so that the global aspects of the problem remained virtually identical to those in pure Einstein gravity. It is tempting to think that this may exemplify a more general connection between the global properties of the (space of) classical solutions and the qualitative behavior of thermodynamical equilibrium ensembles: one might conjecture that whenever the global properties of a Lovelock theory are sufficiently similar to those of Einstein's theory, then also the equilibrium thermodynamics, with finite or infinite boundary conditions, will be qualitatively similar to that in Einstein's theory. Another example supporting such a conjecture is provided by the asymptotically anti-de Sitter Lovelock theories of Ref. [47], which include as a special case Einstein gravity in three and four dimensions with a negative cosmological constant. However, to give the conjecture a more substantial meaning, one would need a more systematic understanding of the possible global structures that the various Lovelock theories may have.

\section{ACKNOWLEDGMENTS}

We would like to thank John Friedman, Eric Poisson, and Bernard Whiting for helpful discussions. Some of the results in sections $\mathbb{\square}$ and $\mathrm{D}$ overlap with previous work by one of us (J.Z.S) and Bernard Whiting [44]. Much of the work was done using MATHEMATICA [48] and MATHTENSOR [49]: in particular, the spherically symmetric action (2.2) was derived independently both on a computer and by hand, and we used a computer to verify that the equations of motion derived from (2.2) are equivalent to the correct spherically symmetric equations, derived from (1.1). This work was supported in part by NSF grants PHY-94-13253, PHY-94-21849, and PHY-95-07740. 


\section{APPENDIX A: LOVELOCK BLACK HOLE}

In this appendix we briefly review the classical solutions to our Lovelock theory.

By the Lovelock generalization of Birkhoff's theorem [5], the general solution to the theory (1.1) for $\lambda \neq 0$ can be written in the local curvature coordinates $(T, R)$ as

$$
d s^{2}=-F d T^{2}+F^{-1} d R^{2}+R^{2} d \Omega_{3}^{2}
$$

where

$$
F=1+\frac{R^{2}}{\hat{\lambda}}\left(1 \pm \sqrt{1+\frac{2 \omega \hat{\lambda}}{R^{4}}}\right)
$$

with $\lambda=\frac{1}{2} \hat{\lambda}$. The coordinates $T$ and $R$ are respectively the Killing time, whose constant value hypersurfaces are spacelike or timelike depending on the sign of $F$, and the curvature radius of the three-sphere. For $\lambda=0$, the general solution is the five-dimensional Schwarzschild solution, obtained from (A2) with the lower sign as the limit $\hat{\lambda} \rightarrow 0$. In addition to $\lambda$, the only local parameter in the solution is the mass-like quantity $\omega$.

For the reasons discussed in section $\mathbb{1}$, we take $\hat{\lambda} \geq 0$. The solution then describes a black hole in asymptotically flat space provided we take $\omega>\frac{1}{2} \hat{\lambda}$ and, for $\hat{\lambda}>0$, choose the lower sign in (A2) [11. The curvature coordinates are good individually in each region not containing horizons: the horizons are nondegenerate, and the Penrose diagram of the conventional maximal analytic extension is similar to that of Kruskal manifold. The horizon radius is $R_{h}=\sqrt{\omega-\frac{1}{2} \hat{\lambda}}$. In our units, the ADM mass is $M=\frac{1}{2} \omega$.

\section{APPENDIX B: ASYMPTOTICALLY FLAT INFINITY}

In this appendix we adapt the analysis of the main text to boundary conditions that replace the timelike boundary by an asymptotically flat infinity. We shall see that quantization along the lines of section $[\mathrm{V}$ will not lead to a well-defined canonical ensemble.

In the metric theory of section $\mathbb{1 1}$, we let $r$ take the range $0 \leq r<\infty$. At $r \rightarrow \infty$, we introduce the falloff

$$
\begin{aligned}
\Lambda(t, r) & =1+M_{+}(t) r^{-2}+O^{\infty}\left(r^{-2-\epsilon}\right) \\
R(t, r) & =r+O^{\infty}\left(r^{-1-\epsilon}\right) \\
P_{\Lambda}(t, r) & =O^{\infty}\left(r^{1-\epsilon}\right) \\
P_{R}(t, r) & =O^{\infty}\left(r^{-\epsilon}\right) \\
N(t, r) & =N_{+}(t)+O^{\infty}\left(r^{-\epsilon}\right) \\
N^{r}(t, r) & =O^{\infty}\left(r^{-1-\epsilon}\right)
\end{aligned}
$$

where $N_{+}(t)>0$, and $\epsilon$ is a parameter that can be chosen arbitrarily in the range $0<\epsilon \leq 2$. $O^{\infty}\left(r^{s}\right)$ denotes a term that is bounded at $r \rightarrow \infty$ by a constant times $r^{s}$, and whose 
derivatives fall off accordingly. It is straightforward to verify that this falloff makes the coordinates asymptotic to hyperspherical coordinates in Minkowski space, it is consistent with the constraints, and it is preserved by the time evolution. $N_{+}(t)$ gives the rate at which the asymptotic Minkowski time advances with respect to the coordinate time $t$. When the equations of motion hold, $M_{+}(t)$ is independent of $t$, and its value is the ADM mass.

The total action takes the form $S=S_{\Sigma}+S_{\partial \Sigma}$, where

$$
S_{\partial \Sigma}=\int d t R_{0}\left(\frac{1}{3} R_{0}^{2}+\hat{\lambda}\right)\left(N_{1} / \Lambda_{0}\right)-\int d t N_{+} M_{+}
$$

and $S_{\Sigma}$ is as in (2.4) except that the upper limit of the $r$-integral is replaced by infinity. The canonical transformation and Hamiltonian reduction proceed as in section III. The action of the reduced theory is as in (3.9), but with the Hamiltonian now given by

$$
\mathbf{h}=N_{+} \mathbf{m}-\mathrm{N}_{0} R_{h}\left(\frac{1}{3} R_{h}^{2}+\hat{\lambda}\right) .
$$

The configuration variable $\mathbf{m}$ has the range $\mathbf{m}>\frac{1}{4} \hat{\lambda}$, while the range of $\mathbf{p}$ is the full real axis. Although in the main text we assumed for presentational simplicity $\hat{\lambda}>0$, it is easily verified that the above derivation extends to the case $\hat{\lambda}=0$, and the reduced Hamiltonian (B3) is valid for all $\hat{\lambda} \geq 0$.

Quantization can now proceed as in the main text. We analytically continue the time evolution operator by $\int \mathrm{N}_{0} d t=-2 \pi i$ and $\int N_{+} d t=-i \beta$, interpreting $\beta$ as the inverse temperature at the infinity. For the renormalized trace of the analytically continued time evolution operator, we obtain formally

$$
Z(\beta ; \hat{\lambda})=\mathcal{N} \int_{0}^{\infty} \tilde{\mu} d R_{h} \exp \left(-I_{*}^{\infty}\right)
$$

where the effective action $I_{*}^{\infty}$ is given by

$$
I_{*}^{\infty}=\frac{1}{2} \beta\left(R_{h}^{2}+\frac{1}{2} \hat{\lambda}\right)-2 \pi R_{h}\left(\frac{1}{3} R_{h}^{2}+\hat{\lambda}\right) .
$$

The smooth and slowly varying positive function $\tilde{\mu}\left(R_{h}\right)$ arose from the choice of the inner product, and $\mathcal{N}$ is a normalization constant, possibly dependent on $\hat{\lambda}$ but presumably not on $\beta$. However, the integral in ( $\mathrm{B} 4)$ is divergent because $I_{*}^{\infty}$ tends to $-\infty$ at large $R_{h}$. Thus, the canonical ensemble does not exist under the asymptotically flat boundary conditions, neither for $\hat{\lambda}=0$ nor $\hat{\lambda}>0$. In this sense, the asymptotically flat Lovelock theory is thermodynamically no better behaved than asymptotically flat Einstein theory.

The critical points of $I_{*}^{\infty}$ give the (Lorentzian) classical solutions that have the inverse Hawking temperature $\beta$ at infinity. For $\hat{\lambda}=0$ there exists exactly one critical point, which is a local maximum: this is similar to what happens with Einstein's theory in four dimensions [36. For $\hat{\lambda}>0$, the situation is more versatile. Critical points exist when $\beta^{2} \geq 16 \pi^{2} \hat{\lambda}$, and when the inequality is genuine, there are two critical points. The critical point with the smaller (larger) value of $R_{h}$ is a local minimum (maximum, respectively). The local minimum gives the classical solution that was found to be stable against Hawking evaporation in Ref. [11]. While the stability of this solution against Hawking evaporation reflects its being a local minimum of $I_{*}^{\infty}$ [38], the divergence of the integral in (B4) demonstrates that this local 
stability is not sufficient to guarantee the existence of the canonical ensemble. The effects of the Lovelock parameter on the asymptotically flat thermodynamics are thus qualitatively very similar to those of a fixed charge in asymptotically flat four-dimensional EinsteinMaxwell theory [24,50,51]. 


\section{REFERENCES}

[1] D. Lovelock, J. Math. Phys. 12, 498 (1971).

[2] D. Lovelock, J. Math. Phys. 13, 874 (1972).

[3] D. G. Boulware and S. Deser, Phys. Rev. Lett. 55, 2656 (1985); Phys. Lett. 175B, 409 (1986).

[4] J. T. Wheeler, Nucl. Phys. B268, 737 (1986); B273, 732 (1986).

[5] D. L. Wiltshire, Phys. Lett. 169B, 36 (1986).

[6] R. C. Myers, Phys. Rev. D 36, 392 (1987). [Equation (7) is in error; see Ref. [13].]

[7] C. Teitelboim and J. Zanelli, Class. Quantum Grav. 4, L125 (1987).

[8] C. Teitelboim and J. Zanelli, in Constraints Theory and Relativistic Dynamics, edited by G. Longhi and L. Lusanna (World Scientific, Singapore, 1987).

[9] D. L. Wiltshire, Phys. Rev. D 38, 2445 (1988).

[10] B. Whitt, Phys. Rev. D 38, 3000 (1988).

[11] R. C. Myers and J. Z. Simon, Phys. Rev. D 38, 2434 (1988).

[12] R. C. Myers and J. Z. Simon, Gen. Relativ. Gravit. 21, 761 (1989).

[13] J. Melmed, Ph.D. Thesis, University of North Carolina, Chapel Hill (1989, unpublished).

[14] E. Poisson, Class. Quantum Grav. 8, 639 (1991).

[15] E. Poisson, Phys. Rev. D 43, 3923 (1991).

[16] K. Maeda, Phys. Lett. 166B, 59 (1986); J. Madore, Phys. Lett. 110A, 289 (1985); Phys. Lett. 111A, 283 (1985); Class. Quantum Grav. 3, 361 (1986); N. Deruelle and J. Madore, Phys. Lett. 114A, 185 (1986); Phys. Lett. 186B, 25 (1987); H. Ishihara, Phys. Lett. 179B, 217 (1986); W. Puszkarz, Phys. Lett. 226B, 39 (1989); N. Deruelle and L. Farina-Busto, Phys. Rev. D 41, 3696 (1990).

[17] T. Jacobson and R. C. Myers, Phys. Rev. Lett. 70, 3684 (1993). (hep-th/9305016)

[18] T. Jacobson, G. Kang, and R. C. Myers, Phys. Rev. D 49, 6587 (1994). (gr-qc/9312023)

[19] T. Jacobson, G. Kang, and R. C. Myers, Phys. Rev. D 52, 3518 (1995). (gr-qc/9503020).

[20] K. V. Kuchař, Phys. Rev. D 50, 3961 (1994). (gr-qc/9403003)

[21] J. Louko and B. F. Whiting, Phys. Rev. D 51, 5583 (1995). (gr-qc/9411017)

[22] G. Oliveira-Neto, Phys. Rev. D 53, 1977 (1996).

[23] S. R. Lau, Class. Quantum Grav. 13, 1541 (1996). (gr-qc/9508028)

[24] J. Louko and S. N. Winters-Hilt, Phys. Rev. D 54, 2647 (1996). (gr-qc/9602003)

[25] M. Varadarajan, Phys. Rev. D 52, 7080 (1995). (gr-qc/9508039)

[26] S. Bose, J. Louko, L. Parker, and Y. Peleg, Phys. Rev. D 53, 5708 (1996). (grqc/9510048)

[27] J. D. Romano and C. G. Torre, Phys. Rev. D 53, 5634 (1996). gr-qc/9509055)

[28] K. V. Kuchař, J. D. Romano, and M. Varadarajan, "Dirac Constraint Quantization of a Dilatonic Model of Gravitational Collapse", e-print gr-qc/9608011.

[29] T. Thiemann and H. A. Kastrup, Nucl. Phys. B399, 211 (1993). (gr-qc/9310012)

[30] H. A. Kastrup and T. Thiemann, Nucl. Phys. B425, 665 (1994). (gr-qc/9401032)

[31] T. Thiemann, Int. J. Mod. Phys. D 3, 293 (1994).

[32] T. Thiemann, Nucl. Phys. B436, 681 (1995).

[33] J. Gegenberg and G. Kunstatter, Phys. Rev. D 47, R4192 (1993). (gr-qc/9302006)

[34] J. Gegenberg, G. Kunstatter, and D. Louis-Martinez, Phys. Rev. D 51, 1781 (1995). (gr-qc/9408015) 
[35] D. Louis-Martinez and G. Kunstatter, Phys. Rev. D 52, 3494 (1995). (gr-qc/9503016)

[36] J. W. York, Phys. Rev. D 33, 2092 (1986).

[37] B. F. Whiting and J. W. York, Phys. Rev. Lett. 61, 1336 (1988).

[38] B. F. Whiting, Class. Quantum Grav. 7, 15 (1990).

[39] G. W. Gibbons and S. W. Hawking, Phys. Rev. D 15, 2752 (1977).

[40] S. W. Hawking, in General Relativity: An Einstein Centenary Survey, edited by S. W. Hawking and W. Israel (Cambridge University Press, Cambridge, 1979).

[41] J. D. Brown and J. W. York, Phys. Rev. D 47, 1407 (1993).

[42] S. R. Lau, Class. Quantum Grav. 13, 1541 (1996). (gr-qc/9504026)

[43] S. W. Hawking and C. J. Hunter, Class. Quantum Grav. 13, 2735 (1996). (grqc/9603050)

[44] J. Z. Simon and B. F. Whiting (unpublished).

[45] H. W. Braden, B. F. Whiting, and J. W. York, Phys. Rev. D 36, 3614 (1987).

[46] J. Z. Simon, Phys. Rev. D 41, 3720 (1990); Phys. Rev. D 43, 3308 (1991); Phys. Rev. D 45, 1953 (1992); L. Parker and J. Z. Simon, Phys. Rev. D 47, 1339 (1993).

[47] M. Bañados, C. Teitelboim, and J. Zanelli, Phys. Rev. D 49, 975 (1994). (gr-qc/9307033)

[48] Wolfram Research, Inc., MATHEMATICA (Wolfram Research, Inc., Champaign, IL, 1992).

[49] L. Parker and S. M. Christensen, MATHTENSOR (MathSolutions, Inc., Chapel Hill, NC, 1992).

[50] P. C. W. Davies, Proc. R. Soc. London A353, 499 (1977).

[51] P. C. W. Davies, Rep. Prog. Phys. 41, 1313 (1978). 